\title{
A Note on the Interpretation of Scale Values in Multidimensional Scaling Growth Analysis
}

\author{
Cody Ding \\ University of Missouri-St. Louis
}

\begin{abstract}
Multidimensional Scaling (MDS) has been used as a growth mixture modeling technique in psychological and education research in recent years. This note focuses on a detailed explanation of interpreting the scale values in MDS growth analysis. Since scale values from MDS growth analysis are based on the Euclidean metric, we attempt to offer some guidance on interpretation of the scale values in terms of percentage of change in growth between each time interval. This approach is illustrated with a hypothetical example, and it can be used in actual research settings.
\end{abstract}

Keywords: multidimensional scaling, growth analysis, scale values, percentage of change

During recent decades, researchers have witnessed significant progress in growth modeling techniques, from both theoretical and data-analytical perspectives. The newer statistical models include latent growth model (e.g., Collins \& Sayer, 2001), finite growth mixture model (e.g., Muthen \& Muthen, 2000), and multilevel linear model (e.g., Little, Schnabel, \& Baumert, 2000; Singer \& Willett, 2003) for the analysis of longitudinal data in education and psychology. Today, these methodologies are commonly applied when studying growth and change of human behaviors.

In the current literature, growth mixture modeling represents the most common method of dealing with latent growth classes because it provides a way to empirically identify the number of latent classes in a population and to estimate the growth trajectory within each latent class. A less wellknown growth mixture modeling is the multidimensional scaling (MDS) latent growth profile analysis that also allows researchers to estimate latent trajectory classes and to study their relations to either covariates or outcome measures (Ding, Davison, \& Petersen, 2005). Conceptually, the MDS latent growth model has similar analytic goals as growth mixture models (GMM) or the group-based approach (Nagin, 1999)--to determine the optimal number of latent growth groups and the shape of the trajectory that best fits the data for each group. Then, outcome measures and covariates can be incorporated into the analysis with respect to the different latent growth groups. The MDS model differs, however, from GMM or group-based approaches in terms of how to present growth rates, identifying the latent groups, and the assumptions underlying its use. Briefly, a latent group in the MDS model is called a "latent growth profile", 
which is represented by a single dimension. The dimension is estimated from a distance model and consists of a set of scale values that indicate the shape of the growth trajectory. A hypothetical example of growth patterns in student mathematical achievement is shown in Figure 1, where Profile 1 indicates a linear growth and Profile 2 indicates a down-up growth. Each profile represents a subgroup of individuals who manifest a particular growth trajectory in mathematic achievement. The use of dimension as an indication of latent group presence has been studied by Davison and colleagues (Davison, Gasser, \& Ding, 1996; Davison, Kuang, \& Kim, 1999; Kim, Davison, \& Frisby, 2007).

In MDS growth analysis, MDS is based on distance models, and the distance between any pair of adjacent time points $\left(t_{\mathrm{j}}\right.$ and $\left.t_{\mathrm{j}+1}\right)$ represents a growth rate for that time interval, as shown in Figure 1 . That is, the differences between scale values of adjacent time points indicate the change (i.e., slope) for a given time interval, as shown in Figure 1. That is, the differences between scale values of adjacent time points indicates the change (i.e., slope) for a given time interval, and the time intervals do not need to be evenly spaced; the growth rate is the slope for each particular interval. If the time unit between time 1 and time 2 is one month but the time unit between time 3 and time 4 is one year, then slope indicates $_{1}$ growth for one month and slope ${ }_{2}$ is the growth for one year. Moreover, measurement units over time do not need to be in the same metric. The reason for no such requirement is that the growth rate is based on the scale value in MDS, which has the common Euclidean metric over time. Put another way, a scale value of, say, 1.4 at time $t$ has the same meaning for a scale value of, say, 2.6 at time $t+1$, which indicate an equivalent distance between two time points, as shown in Figure 1, regardless of whether time interval is equal or unequal. Thus, a particular set of scale values in MDS growth analysis indicates the changes over time for a specific behavior during a particular time frame.

The overall rational for use of MDS model in growth analysis has been articulated in previous research (e.g., Ding, et al., 2005). In this methodological note we focus on an explanation in more detail, of the interpretation of the scale values of the MDS growth analysis so that researchers or practitioners may have a better grasp on the issue. MDS scale values are difficult to interpret because they have no upper or lower bound and can range from zero to $\pm \infty$. In our example, the interpretation of scale values of 1.4 at time $t$ and of 2.6 at time $t+1$, is not quite intuitive and it is hard to evaluate the magnitude of change. All we know is that the change is positive (i.e., growth), with a difference of 1.2 in scale values between these two time points. Therefore, it would be good if we have a familiar metric that can be used to evaluate magnitude of change based on scale values. In this note, we discuss the percentage of change in scale value as a metric to evaluate the magnitude of change. 
Since the scale values in the spatial distance model are of Euclidean space, indicating distance between two points, they satisfy the following axioms (Davison, 1983):

$$
\begin{aligned}
& d(a, b) \geq 0, \\
& d(a, a)=0, \\
& d(a, b)=d(b, a)
\end{aligned}
$$

where $d$ represents distance between points $a$ and $b$. Thus, we can assume that when the scale values between any pair of time points are equal, they indicate no change. On the other hand, when there is a difference in scale values between any pair of time points, it indicates that there may be a change in the behavior under inquiry.

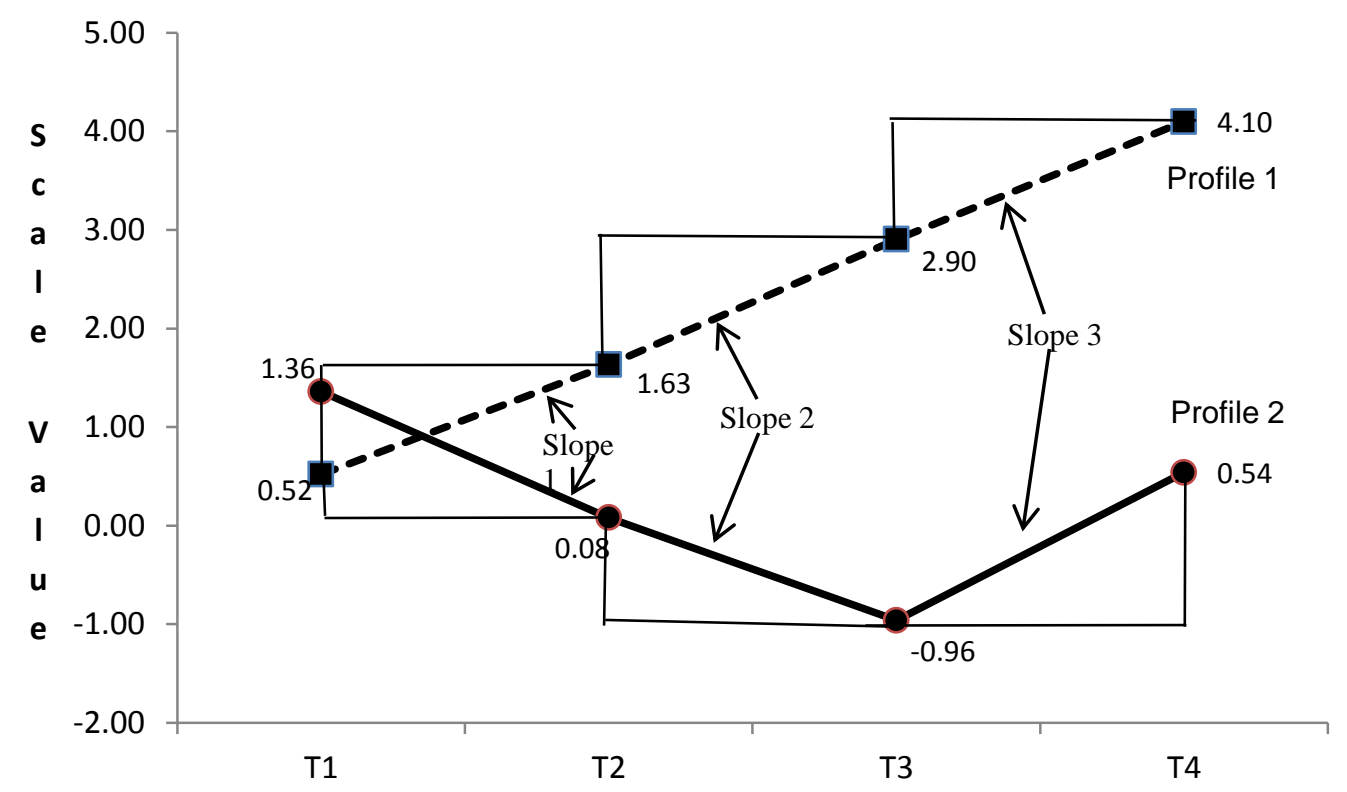

Figure 1. A hypothetical example of growth profiles of student mathematic achievement in a two-dimensional solution of MDS growth analysis.

Table 1 shows the scale values of two growth profiles (i.e., a twodimensional solution) depicted in Figure 1. In an attempt to offer some guidance to researchers on interpretation of scale values that reflect changes over a particular time period in a two-dimensional solution, we can calculate the percentage of change in scale values between any pair of time points in the following fashion:

1. Calculate the difference in scale values between time $t$ and time $t+1$ for each time interval. In our hypothetical example, a set of three difference scores, one for each time interval, is calculated for each growth profile, as shown in the middle part of Table 1. 
2. Calculate the total change in scale values over the entire time period under study using the absolute difference in scale values since the sign only indicates direction of change. This is a total change score for each profile.

3. Calculate the percentage of change for each time interval by dividing the difference score by the total change score. The resulting number indicates the percentage of change with respect to the total amount of change, as shown in the lower part of Table 1.

Thus, based on these calculations for our hypothetical example of growth profiles, we can say that for Profile 1, there is a $31 \%$ change from time 1 to time 2 , a $36 \%$ change from time 2 to time 3 , and a $33 \%$ change from time 3 to time 4 . The overall change for individuals who resemble Profile 1 is positive, with an average of $33 \%$ change over time, assuming time 1 is the initial time point. Similarly, for Profile 2, there is a negative change of $34 \%$ from time 1 to time 2, a negative change of $27 \%$ from time 2 to time 3 , and a positive change of $39 \%$ from time 3 to time 4 . The overall change for individuals who resemble Profile 2 is negative, with an average of negative $7 \%$ change over time. Although there is an increase from time 3 to time 4 , such an increase is not large enough to counterbalance the impact of negative change.

Table 1

Scale values, difference scores, and percentage of change in MDS growth analysis

\begin{tabular}{ccc}
\hline Time & Profile 1 & Profile 2 \\
\hline T1 & 0.52 & 1.36 \\
T2 & 1.63 & 0.08 \\
T3 & 2.90 & -0.96 \\
T4 & 4.10 & 0.54 \\
\hline \multicolumn{3}{c}{ Difference in Scale Values } \\
T2 - T2 & 1.11 & -1.28 \\
T4 - T3 & 1.27 & -1.04 \\
Total Change & 1.20 & 1.50 \\
\hline & 3.58 & 3.82 \\
\hline T1 to T2 & \% of Change \\
T2 to T3 & 0.31 & -0.34 \\
T3 to T4 & 0.36 & -0.27 \\
\hline
\end{tabular}

Note: Total change score is based on the absolute difference in scale values between two time points. A negative sign only indicates the direction of change. 
In conclusion, this note offers, in more detail, a method for calculation of growth rate in terms of percentage based on scale values in MDS growth analysis. The percentage changes are easier to understand than other types of scores such as z-scores. Although the method is not very sophisticated in terms of novelty, it has the advantages of being (1) simple and (2) more intuitive in interpretation of the magnitude of change over time. The hypothetical example is realistic enough to offer researchers some useful guidance on how to determine the magnitude of change between each time interval and of the overall change rate. Note that the percentage of change for each time interval is interpreted only with respect to the total amount of change in scale values rather than to the absolute change in behaviors. It is recommended to convert scale values in MDS growth analysis into percentage of changes as a metric for interpretation.

Corresponding author: Cody Ding, 404 Marillac Hall, University of MissouriSt. Louis, St. Louis, MO 63121. email: dingc@umsl.edu

\section{References}

Collins, L. M., \& Sayer, A. G. (Eds.). (2001). New methods for the analysis of change. Washington, DC: American Psychological Association.

Davison, M. L. (1983). Multidimensional scaling. New York: Wiley.

Davison, M. L., Gasser, M., \& Ding, S. (1996). Identifying major profile patterns in a population: An exploratory study of WAIS and GATB patterns. Psychological Assessment, 8, 26-31.

Davison, M. L., Kuang, H., \& Kim, S. (1999). The structure of ability profile patterns: A multidimensional scaling perspective on the structure of intellect. In P. L. Ackerman, K. P. C. \& R. R.D. (Eds.), Learning and individual differences: Process, trait, and content determinants (pp. 187 - 204). Washington, D. C.: APA Books.

Ding, C. S., Davison, M. L., \& Petersen, A. C. (2005). Multidimensional scaling analysis of growth and change. Journal of Educational Measurement, 42, 171-191.

Kim, S., Davison, M. L., \& Frisby, C. L. (2007). Confirmatory factor analysis and profile analysis via multidimensional scaling. Multivariate Behavioral Research, 42, 1-32.

Little, T. D., Schnabel, K. U., \& Baumert, J. (2000). Modeling longitudinal and multilevel data. Mahwah, NJ: Lawrence Erlbaum Associates.

Muthen, B. O., \& Muthen, L. K. (2000). Integrating person-centered and variable-centered analyses: Growth mixture modeling with latent trajectory classes. Alcoholism: Clinical and Experimental Research, 24, 882-891.

Nagin, D. (1999). Analyzing developmental trajectories: A semi-parametric, group-based approach. Psychological Methods, 4, 139-177.

Singer, J. D., \& Willett, J. B. (2003). Applied longitudinal data analysis. New York, NY: Oxford University Press. 